\title{
Reflection and refraction of an extreme ultraviolet wave at boundary of coronal hole
}

\author{
Zhike Xue ${ }^{1}$, Zhongquan Qu ${ }^{1}$, Xiaoli Yan ${ }^{1}$, and Li Zhao ${ }^{2}$ \\ ${ }^{1}$ National Astronomical Observatories of China/Yunnan Astronomical Observatory, \\ Chinese Academy of Sciences, Kunming, Yunnan 650011, China \\ ${ }^{2}$ Yunnan Normal University, Kunming 650092, China
}

\begin{abstract}
We present reflection and refraction of a global EUV wave observed by SDO on August 4, 2011. The global EUV wave originated in the NOAA AR 11261 located at the solar northwest. Partial EUV wave stopped and partial EUV wave traversed through the active region 11263 during the propagation of the EUV wave. Partial EUV wave interacted with coronal hole and was reflected and refracted by coronal hole.
\end{abstract}

Keywords. Sun: activity, Sun: sunspots, Sun: corona

\section{Introduction}

Extreme Ultraviolet (EUV) waves were first reported by Moses et al. (1997) and Thompson et al. (1998) with the EIT on board SOHO. When EUV waves interact with active regions (ARs) and/or coronal holes (CHs), refraction or reflection always happens. Ofman \& Thompson (2002) found strong refraction and reflection from an AR. Gopalswamy et al. (2009) reported wave reflection from a small CH. EUV wave deflection was observed by Olmedo et al. (2012). They showed that part of EUV wave transmitted through the $\mathrm{CH}$. These phenomena indicate that EUV waves are true waves.

\section{Results}

On 2011 August 4, a global EUV wave was observed by SDO/AIA in association with an M9.3 flare, a filament eruption and a CME. The EUV wave was observed most clearly in the AIA $193 \AA$ channel. The global EUV wave initiated from NOAA AR 11261 with a dome-shaped structure at 03:49 UT. When the wave front arrived at the NOAA AR 11263, the wave along the path S1 splits two parts (see Figure 1c). One part (W1) propagated along the original direction with a constant speed. The other part (W2) slowed down and disappeared. A coronal hole located in the southern hemisphere (Figure 1a). A
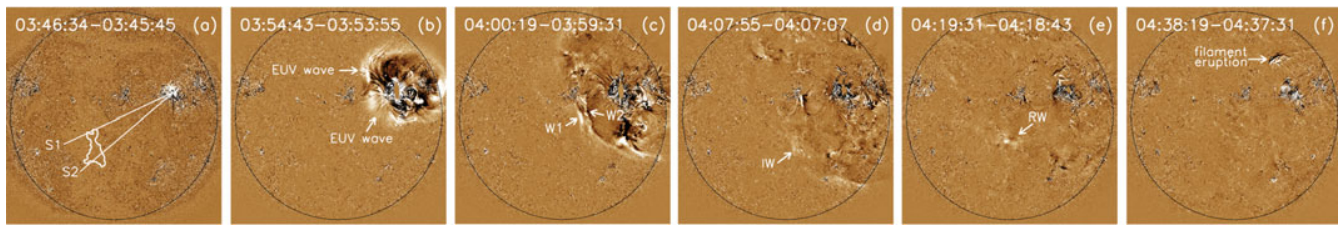

Figure 1. The running difference images of the coronal EUV wave observed with AIA $193 \AA$ channel. The slice S1 passed through the AR 11263 and slice S2 was along the propagation paths of the incident and reflected wave. W1 and W2 is two split parts of the EUV wave. IW is the incident wave and RW represents the reflected wave. The white curved line in panel (a) shows the position of coronal hole on the solar disk. 
reflected wave (RW) was observed after the incident wave (IW) reached the boundary of the $\mathrm{CH}$ along the path S2 (Figure 1e). Then the reflected EUV wave was scattered and disappeared gradually. In order to study this EUV wave quantitatively, the propagation speeds along two paths (S1 and S2) were obtained with running difference time-slice images (Figure 2a and b). In Figure 2a the two parts of the wave were recognized easily (Figure 2a). The wave speed was $332 \pm 16 \mathrm{~km} \mathrm{~s}^{-1}$ along the slice S1. Form Figure 2b, one can find that the wave was reflected back when it contacted the $\mathrm{CH}$. The speeds of incident and reflected wave were $320 \pm 31 \mathrm{~km} \mathrm{~s}^{-1}$ and $315 \pm 38 \mathrm{~km} \mathrm{~s}^{-1}$ respectively. In order to research the interaction between the wave and the $\mathrm{CH}$, the boundaries of the $\mathrm{CH}$ were shown in Figure 3 with the white solid lines. It was obtained from $193 \AA$ images along S2. The boundary of the $\mathrm{CH}$ on the northwest side shrank back after the EUV wave arrived. Then it expanded gradually. However, the other boundary of the $\mathrm{CH}$ almost unchanged.

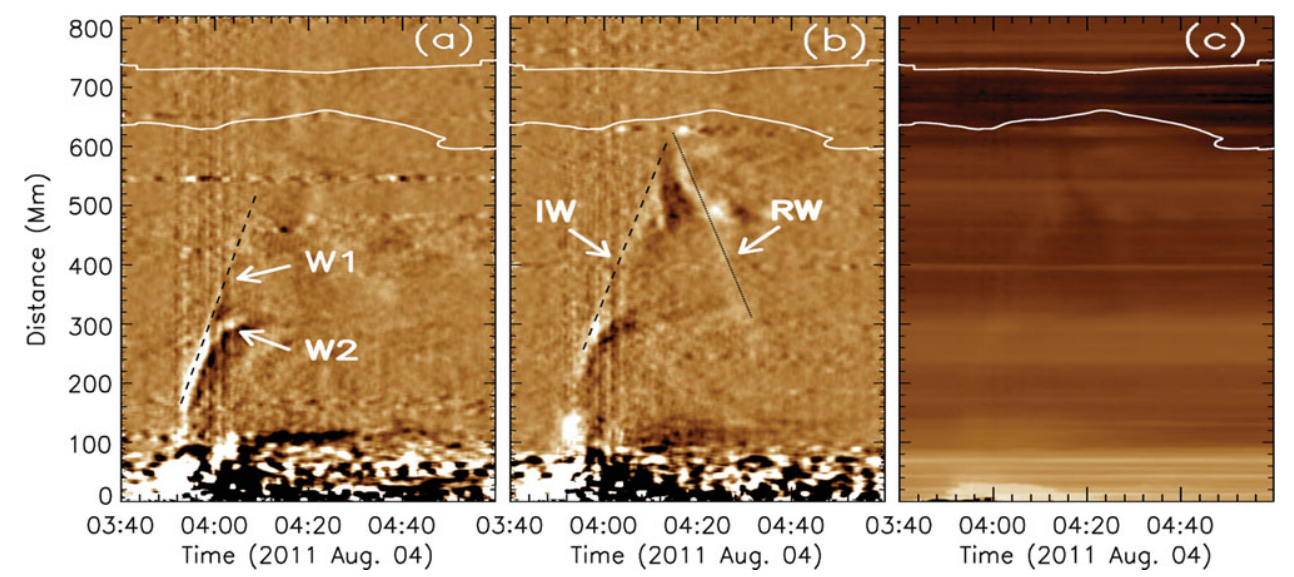

Figure 2. Panel (a) and (b) show running difference time-slice images along S1 and S2. Panel (C) shows the time-slice images along the slice S2 obtained from AIA $193 \AA$ images. The white lines represent the coronal hole boundaries obtained from panel (c).

\section{Discussions and Conclusion}

The topic for that EUV waves are true wave or not is discussed for many years. If they are true wave, they must accord with characters of waves. The refraction and reflection of wave are perfect evidences. Here we observed the reflection that it to some extent indicates that the EUV wave is true wave. The speed of incident wave is similar to that of reflect wave. Contrasting with the boundary changes of other positions of the $\mathrm{CH}$, we conclude that the change of the $\mathrm{CH}$ boundary may be that the EUV wave transports into the $\mathrm{CH}$, rather than real $\mathrm{CH}$ changes. This implies the refraction happened.

\section{References}

Gopalswamy N., Yashiro S., Temmer M. et al. 2009, ApJ, 691, L123

Moses, D., Clette, F., Delaboudiniere, J.-P., et al. 1997, Sol. Phys., 175, 571

Ofman, L. \& Thompson, B. J. 2002, ApJ, 574, 440

Olmedo, O., Vourlidas, A., Zhang, J., et al. 2012, arXiv:1206.6137v1

Thompson, B. J., Plunkett, S. P., Gurman, J. B., et al. 1998, Geophys. Res. Lett., 25, 2465 